\title{
Corporación nacional de telecomunicaciones - CNT EP: Análisis de gestión pública en la sociedad ecuatoriana
}

\section{National telecommunications corporation - CNT EP: Public management analysis in Ecuadorian society}

\author{
Maryury Elizabeth Morejón-Santistevan \\ mayuri.morejon@unesum.edu.ec \\ Universidad Estatal del Sur de Manabí \\ Ecuador \\ https://orcid.org/0000-0002-2180-7642
}

Recibido: 23 de mayo de 2019

Aprobado: 24 de junio de 2019

\begin{abstract}
RESUMEN
La investigación tuvo por objetivo analizar a la corporación nacional de telecomunicaciones - CNT EP y su incidencia en la gestión pública en la sociedad ecuatoriana. Metodológicamente se sustentó desde la investigación descriptiva no experimental, con una población de 516 personas. Entre las principales conclusiones se tiene la empresa se encuentra desarrollándose en conformidad con los postulados de su filosofía de gestión y con la nueva gestión pública (NGP), lo cual permite proyectar el funcionamiento de una empresa pública en similitud de las operaciones de una privada, siendo esto un beneficio para la sociedad, observándose un eficiente aporte a la gestión pública del Ecuador.
\end{abstract}

Descriptores: Responsabilidad social; Gobernabilidad; Administración pública; Empresa pública.

\footnotetext{
ABSTRACT

The objective of the research was to analyze the national telecommunications corporation - CNT EP and its impact on public management in Ecuadorian society. Methodologically it was supported from non-experimental descriptive research, with a population of 516 people. Among the main conclusions is the company is being developed in accordance with the postulates of its management philosophy and the new public management (NGP),
} 


\section{CIENCIAMATRIA \\ Revista Interdisciplinaria de Humanidades, Educación, Ciencia y Tecnología \\ Año VI. Vol. VI. N¹0. Enero - Julio 2020 \\ Hecho el depósito de ley: pp201602FA4721 \\ ISSN-L: 2542-3029; ISSN: 2610-802X \\ Universidad Nacional Experimental Francisco de Miranda (UNEFM). Santa Ana de Coro. Venezuela}

Maryuri Elizabeth Morejón Santistevan

which allows to project the operation of a public company similar to the operations of a private company, this being a benefit for society, observing an efficient contribution to the public management of

Ecuador.

Descriptors: Social responsibility; Governance; Public administration; Public enterprises.

\section{INTRODUCCIÓN}

Se ha venido comentando en el ámbito de la administración pública de los últimos años, la perspectiva de la nueva gerencia pública (NGP), en la gestión pública, lo cual ha permitido a las empresas del Estado, modernizarse y acercarse a la prestación de servicios en competencia con la empresa privada. En el Ecuador se ha venido asumiendo progresivamente esta postura empresarial, en específico en lo relacionado al sector de telecomunicaciones, lo cual hace necesario analizar a la corporación nacional de telecomunicaciones - CNT EP y su incidencia en la gestión pública en la sociedad ecuatoriana, esto con el propósito de establecer una reflexión sobre los avances que se ha tenido en compaginar la NGP en la industria ecuatoriana. En tal sentido, la Corporación nacional de telecomunicaciones - CNT EP (2017), se define como:

La Corporación Nacional de Telecomunicaciones (CNT EP) es la empresa pública de telecomunicaciones del Ecuador, creada como resultado de la fusión en 2008 de las empresas ANDINATEL S.A. y PACIFICTEL S.A. y, posteriormente en 2010, de la absorción de la empresa TELECSA. Con presencia en las 24 provincias del país, la Corporación cubría a finales de 2017, 212 de los 221 La CNT EP actúa con una clara estrategia empresa-rial que busca generar rentabilidad económica del negocio que promueve y, a la vez, un importante impacto social, al aportar al presupuesto general del Estado y contemplar requerimientos de in-versión para generar mayores recursos. Asimismo, por su carácter público, proporciona servicios de telecomunicaciones a nivel país, incluyendo a los lugares más apartados del territorio nacional, favoreciendo de manera efectiva la disminución de la brecha digital en el Ecuador. GRI 102-1, 102-2, 102-4CNT7 103-2,103-3 cantones que componen el territorio nacional (p. 8).

Teniendo además en su filosofía de gestión, la visión de ser una empresa comprometida con el impacto social, en correspondencia con brindar servicios de calidad al cliente, trabajar en equipo, ser eficiente, innovar, actuación integra y compromiso social. Estas 


\section{CIENCIAMATRIA}

Revista Interdisciplinaria de Humanidades, Educación, Ciencia y Tecnología

Año VI. Vol. VI. N¹0. Enero - Julio 2020

Hecho el depósito de ley: pp201602FA4721

ISSN-L: 2542-3029; ISSN: 2610-802X

Universidad Nacional Experimental Francisco de Miranda (UNEFM). Santa Ana de Coro. Venezuela

Maryuri Elizabeth Morejón Santistevan

premisas destacan en la posibilidad de que la Corporación nacional de telecomunicaciones - CNT EP, se constituya en una empresa con enfoque que procura brindar calidad, situación que la asemeja a una empresa privada, encontrándose inmersa en el paradigma de nueva gerencia pública (NGP), en la gestión pública. En tal sentido, Pliscoff -Varas (2017), plantea la existencia de tres enfoques políticos de la NGP:

1. Según el modelo propuesto por Kooiman, la gobernanza es entendida como un fenómeno social que cuenta con la participación del gobierno y del sector privado. En este sentido, se entiende el gobierno interactivo como "los acuerdos con los que tanto los actores públicos como los privados persiguen solventar problemas sociales o crear oportunidades sociales". Así, las fronteras divisorias entre el sector público y el privado se están difuminando cada vez más.

2. Otra teoría que se ha planteado en el último tiempo y que ayuda a dar cuenta de uno de los aspectos más débiles de la NGP es el llamado New Public Service (NPS) o Nuevo Servicio Público. Esta aproximación teórica desarrollada por Denhardt y Denhardt, se plantea como una visión diferente a dos extremos, la administración pública tradicional o progresiva y la NGP.

3. La Administración Pública Posmoderna surge como la antítesis al positivismo rechazando los principales supuestos epistemológicos sobre el comportamiento en las ciencias sociales. La corriente de la Administración Pública Posmoderna se basa en las diferencias entre la modernidad, donde el conocimiento se encontraba basado en la razón, y la propia posmodernidad, la razón lógica, pierde la supremacía. Los posmodernos describen la vida como una hiperrealidad, en la cual ocurre un proceso de destrucción de los significados.

La Corporación nacional de telecomunicaciones - CNT EP, podría indicarse que se encuentra en un hibrido entre el punto 1 y 3 , donde se promueve una visión accionaria de transformaciones políticas y del abordaje con la sociedad de la empresa, en este sentido, 
Bedón hizo referencia a los cambios acontecidos en el Ecuador desde 2007 y destacó que gracias al socialismo del Buen Vivir se ha logrado enrumbar al país a través de una adecuada planificación, además de haber recuperado las capacidades y facultades estatales: "en este camino de construcción se creó un Estado distinto, rescatando su autoridad y ámbito de acción, colocando a la planificación participativa y descentralizada como piedra angular de las políticas públicas, dotándole de una nueva institucionalidad y de credibilidad" (información tomada de http://www.planificacion.gob.ec/la-gestion-publicatiene-como-referente-al-buen-vivir/)

La credibilidad es de suma importancia para que la gestión pública pueda calar en el pueblo desde una visión de eficiencia, generándose la posibilidad de promover un accionar que permita prestar servicios eficientes en la medida que se reinvierte en la producción de la empresa, así desde el planteamiento del buen vivir, se regenera el aparato productivo público del Ecuador, contribuyendo a una gestión pública en pertinencia con los retos sociales que se ha planteado el Estado como paradigma social - económico del buen vivir.

\section{REFERENTES TEORICOS}

\section{Nueva gerencia pública (NGP),}

En este sentido, Lane (s/f), citado por Caamaño (2002) expone que:

La corriente de la Nueva Gestión Pública o modernización del sector público, está encaminada a insertar en la organización pública instrumentos similares al mercado como son la licitación, concurso público, las subastas y la contratación externa para las adquisiciones de bienes y servicios por parte de los gobiernos (p. 7).

Esta visión le permite a lo público, contar con herramientas que le permiten generar transparencia en la inversión y gasto generado por las empresas públicas, fomentándose indicadores de competitividad a la hora de adjudicar un determinado contrato. Así mismo,

La Nueva Gerencia Pública es la teoría que transforma la contratación en el medio de comunicación del sector público. Crea un Estado contratista, en el que los recursos serán administrados por medio de una serie de contratos que 
cubrirán los objetivos y las tareas del gobierno en materia de adquisición de bienes y prestación de servicios (Pichardo, 2004, p. 240).

La contratación genera la posibilidad de organización, planeación, de la empresa pública, permite crear filosofías de gestión organizacional en procura de contribuir al articulado de una perspectiva enmarcada a generar beneficios tanto para la empresa, Estado y sociedad, creándose el marco de responsabilidad social como premisa para concatenar las acciones efectivas que permitan accionar en beneficio del ecosistema social donde se desempeña la empresa. Por otro lado, Schröder (2018), expone la NGP posee las siguientes características:

1. Dirección orientada a la competencia mediante la separación de competencias entre los financiadores y los prestadores de servicios

2. Enfoque en la efectividad, eficiencia y calidad del cumplimiento de tareas

3. Separación de la dirección estratégica (¿QUÉ?) de la dirección operativa (¿CÓMO?)

4. Un trato fundamentalmente igual de prestadores de servicios particulares como públicos dentro del marco de la prestación de servicios y presupuestos globales

5. Impulso enfocado de la innovación (como parte de la prestación de servicios) gracias a un manejo operativo delegado (no sólo descentralizado).

Lo planteado permite la separación de poder entre el Estado y quienes operan la empresa, siendo esta independiente en su accionar en requerimientos de políticas y metas aceptadas por el propio Estado, así este toma la posición de gerente de los procesos que demarcan el accionar de la empresa, así mismo, Schröder (2018), comenta que los instrumentos de la Nueva Gestión Pública:

1. Gestión contractual: Hasta ahora la conducción de la administración pública se ha caracterizado por decisiones basadas en el poder jerárquico y por instrucciones individuales. Con la gestión contractual esto se modifica de tal manera, que aquí ya no se dirigirá mediante instrucciones individuales o intervenciones directas, sino 
Universidad Nacional Experimental Francisco de Miranda (UNEFM). Santa Ana de Coro. Venezuela

Maryuri Elizabeth Morejón Santistevan

a través de objetivos acordados sobre la prestación de servicios y costos. Bajo gestión contractual se entiende la dirección de la administración con base en objetivos acordados, que abarca desde la definición de éstos hasta el control de su cumplimiento. La gestión contractual se basa en contratos, es decir, en acuerdos entre las partes contractuales. Ahora bien, ¿quiénes son estas partes contractuales? Por un lado tenemos a la dirección política y por el otro a los prestadores de servicios, es decir, el lado operativo.

2. Transferencia de la responsabilidad sobre los recursos: El principal objetivo de la gestión de contratos es el de enfocar el interés y la atención de las áreas especializadas sobre el rendimiento de su servicio y/o producto. Técnicamente, esto podrá lograrse de la siguiente manera: los servicios que deberán ser prestados a través de las áreas especializadas (productos) son definidos claramente. Para poder generarlos, el área especializada recibirá un presupuesto relacionado con los productos, del cual deberá realizar los pagos delos servicios requeridos para la elaboración del producto. Aquellos servicios que deberán ser adquiridos a través de otros planos organizativos de la administración, siempre serán remunerables. Para poder captar eficientemente la responsabilidad de los recursos, en principio se les deberá transferir a las áreas especializadas las tareas de gestión, dirección y controlling:

1. Organización y disposición del personal

2. Abastecimiento de información y automatización

3. Planeación de presupuestos y recursos

4. Administración de bienes

5. Estimado previo y posterior de los servicios

6. Cálculo de costos y servicios

7. Análisis empresarial de desviaciones

8. Informes 
3. Orientación hacia el rendimiento (output): Una dirección efectiva de la administración sólo es posible desde el output del servicio. Actualmente, éste no es el caso. Por lo general, la dirección de la administración pública se realiza principalmente a través de los insumos (input), es decir, a través de la adjudicación central de recursos. Los presupuestos indican en miles de puntos cuánto dinero podrá ser erogado y de qué manera deberá gastarse este dinero, sin embargo, en ningún lugar dice de manera precisa qué prestaciones de servicios (productos) deberá generar la administración con este dinero y qué resultado podrá esperar realmente el político electo con este presupuesto.

4. Controlling: Como un concepto integral puede definirse como una gestión rentable y efectiva de la administración en el sentido del cumplimiento con las metas preestablecidas. Para tal efecto, el controlling ha de poner a disposición la información necesaria en el momento indicado dentro de los planos de toma de decisiones y el grado de saturación correspondiente. Controlling abarca la totalidad de las funciones que deberán optimizar el suministro de informaciones para las instancias directivas. Al mismo tiempo abarca funciones parciales, como reconocer las necesidades de información, la adquisición de información, la puesta a disposición y aplicación de métodos de análisis y evaluación y la gestión de información preparativa para el control de planeación y resultados. Por consiguiente, controlling representa más que sólo controlar. Es proporcionar la información para poder dirigir un proceso. Dependiendo de la perspectiva del tiempo, del grado de saturación de la información y de la importancia para la comunidad, se diferencia entre controlling estratégico y operativo.

5. Cálculo de costos y prestaciones de servicios Controlling, para ser un real instrumento de apoyo, depende de un cálculo de costos-beneficio (en este caso prestación de servicios).De esta forma se logrará establecer una verdadera relación entre los costos y las prestaciones de servicio, lo cual permitirá a la administración evaluar la rentabilidad de vías alternas para prestar determinado 
servicio. Los actuales sistemas de cálculo dentro de las administraciones públicas, si es que son llevados con seriedad y no utilizados más bien para encubrir gastos no justificables o malversaciones de fondos, no son transparentes en lo que se refiere a la relación entre costos y prestación de servicios. En la mayoría de los casos sólo existe una contabilidad sobre los ingresos y los egresos de dinero. Mediante un cálculo de costo-servicios se crea una referencia en tiempo (período), $y$, con ello también se crea una referencia de eficiencia y por consiguiente, de éxito.

6. Realización de informes: Una parte primordial dentro del concepto de controlling es la realización de informes. La libertad surgida por la descentralización y delegación deberá ser relacionada con la obligación de realizar informes. Aquellos que hayan recibido esta libertad deberán presentar informes a sus delegantes (la administración), detallando el destino de los recursos que les fueron confiados y si realmente han cumplido con los objetivos y con los estándares de calidad establecidos. Quienes redactan la información tienen la obligación de proporcionar los datos relevantes a la administración para que ésta pueda utilizarlos para los fines requeridos. Aquí depende en gran medida de los números de financiamiento y sus sistemas. La mayoría de estas informaciones son almacenadas dentro del cálculo de costos-beneficios. Por tal motivo es importante un apoyo del sistema a través de la contabilidad. Esto significa, que junto con la presentación de informes se implemente el cálculo de costos-beneficio dentro dela administración.

7. Elaboración del presupuesto: El método hasta ahora empleado para planear un presupuesto considera las cifras del año anterior y las nuevas solicitudes de fondos realizadas por las dependencias o unidades productivas. Esto ocasiona que las innovaciones no sean tenidas en cuenta en la administración de los fondos y que debido a que no existen objetivos establecidos, al haber menos presupuesto, se reduzcan los suministros. La elaboración del presupuesto dentro del marco de la Nueva Gestión Pública parte de un proceso de contraflujo. De esta manera, la 


\section{CIENCIAMATRIA}

Revista Interdisciplinaria de Humanidades, Educación, Ciencia y Tecnología

Año VI. Vol. VI. N¹0. Enero - Julio 2020

Hecho el depósito de ley: pp201602FA4721

ISSN-L: 2542-3029; ISSN: 2610-802X

Universidad Nacional Experimental Francisco de Miranda (UNEFM). Santa Ana de Coro. Venezuela

Maryuri Elizabeth Morejón Santistevan

política establece valores límite a través de los cuales la administración directiva establece el presupuesto general.

8. Orientación hacia los ciudadanos/clientes: Un enunciado fundamental de la Nueva Gestión Pública expresa: "Todo aquello que no sirve al ciudadano representa un derroche. "Con ello se expresa que la administración no es un fin en sí y que su única tarea es la de proporcionar los servicios a los que el ciudadano tiene derecho. En algunos países se ha desarrollado una denominada Citizen Charta que compendia los derechos que los ciudadanos pueden exigir al gobierno a cambio del pago de sus impuestos. Entonces el ciudadano ya no es visto como un súbdito, sino que es un cliente que a cambio de sus contribuciones exige el suministro de un cierto número de servicios que tengan cierta calidad. Aquí el gobierno es visto como una moderna empresa prestadora de servicios, que a veces incluso puede competir con ofertantes particulares. Por otra parte, en ciertas áreas podrá aparecer como ofertante monopolista, pero con el compromiso de optimizar continuamente el suministro y la calidad dentro de un marco deben chmarking junto con otras entidades públicas.

9. Personal: El personal es el factor clave para un proceso de modernización exitoso. La modernización de la administración pública sólo será exitosa si el potencial de los recursos humanos es aprovechado totalmente, o, en caso de que existan deficiencias, tendrá que ser mejorado. En el proceso es necesario involucrar a los empleados, pues de lo contrario sólo se logrará sembrar inseguridad y con frecuencia provocar bloqueos en el comportamiento, lo cual obstaculizaría la reforma. Aquí deberán marcarse oportuna y claramente los objetivos, para que los empleados tengan una idea clara sobre la importancia de la modernización y también para mostrarles los beneficios que obtendrán de la misma.

10. Técnica de información: Los principios de dirección antes mencionados y la reforma de conducción en su totalidad exigen un óptimo sistema de información. La compilación de informaciones y la rápida comunicación, la saturación de la 
cantidad de datos para la conducción y posibilidad desde acceso en las bases de datos para satisfacer las necesidades delos clientes requieren de una red de aparatos de procesamiento de datos, para poder garantizar un trabajo ágil y ante todo confiable. Sin una técnica de información y de comunicación con estructuras entrelazadas de cliente/servidor, no se podrá dirigir a las unidades descentralizadas, ni será posible un manejo satisfactorio de los datos del cliente. Sólo a través de esta tecnología se podrán garantizar variantes tipo one-stopshop5 dentro del contacto con los clientes, ya que de ésta depende de que los empleados tengan los mismos conocimientos que la administración. A través de los manuales digitales de organización se hace posible realizar cualquier tipo de prestación de servicios, dentro del área de administración en cualquier momento, independientemente del tiempo y el lugar

11. Gestión de calidad: Cada producto, incluyendo las prestaciones que lo acompañan, posee muchas características diferenciadoras. Inicialmente se definía calidad como un conjunto de características de un bien material o un servicio, que indicaba su utilidad ("inicio de calidad orientado hacia el producto").Pronto saltó a la vista que existían productos que poseían todas las características planeadas y, aun así, no eran atractivos para el comprador. Al establecer las necesidades dentro del mercado, resulta evidente que todo depende de cómo califica el cliente la calidad del producto. Para la valoración (subjetiva) de los productos por el cliente.

Los pasos de la NPG, permiten estructurar una empresa pública acorde a los requerimientos de una gestión pública en capacidad de ser eficiente desde el punto de vista de los procesos gerenciales que genera similitud al manejo de una empresa priva.

\section{METODOLOGÍA DE LA INVESTIGACIÓN}

Se trabajó con una investigación de tipo descriptiva, definida por Arias (2009) como la investigación que "consiste en la caracterización de un hecho, fenómeno o supo con 
establecer su estructura o comportamiento" (p. 64), realizándose un tratamiento estadístico para interpretar una realidad y cumplir con el objetivo general propuesto al inicio del artículo. Se empleó un diseño no experimental, por cuanto no se manipularon deliberadamente las variables de estudio, sino que se realizó una descripción de los datos obtenidos. Al respecto Hernández, Fernández y Baptista (2008), consideran que en los estudios no experimentales "lo que hacemos es observar fenómenos tal y como se dan en su contexto natural para después analizarlos" (p. 208).

La población de acuerdo a Tamayo y Tamayo (2007), "Es la totalidad del fenómeno a estudiar donde las unidades de población poseen una característica común la cual se estudia y da origen a los datos de la investigación" (p. 114), la cantidad de población estuvo conformada por 516 usuarios de la Corporación nacional de telecomunicaciones - CNT EP, a quienes se les aplicó una encuesta, mediante un instrumento tipo escalamiento de Likert de cinco alternativas de respuestas, aplicándose el coeficiente de Alfa de Cronbach, obteniéndose un resultado de 0,89 , lo cual cataloga al instrumento en un rango de muy alta confiabilidad.

\section{RESULTADOS}

La Corporación nacional de telecomunicaciones - CNT EP y su incidencia en la gestión pública en la sociedad ecuatoriana, se presenta a continuación:

\section{Crecimiento responsable \\ Cuadro 1}

\begin{tabular}{cccccccccc}
\multicolumn{2}{c}{$\begin{array}{c}\text { Excelente } \\
5\end{array}$} & \multicolumn{2}{c}{$\begin{array}{c}\text { Buen } \\
5\end{array}$} & & \multicolumn{2}{c}{ Regular } & \multicolumn{2}{c}{ Deficiente } & \multicolumn{2}{c}{ Muy Deficiente } \\
& & & & & & & & & \\
1 & & & & & \\
\hline FA & $\%$ & FA & $\%$ & FA & $\%$ & FA & $\%$ & FA & $\%$ \\
214 & 33 & 123 & 27 & 97 & 20 & 45 & 13 & 37 & 7 \\
\hline
\end{tabular}

La alternativa excelente crecimiento responsable, posee una representación estadística de $33 \%$, en cuanto a la opción buen crecimiento responsable, obtuvo el $27 \%$, regular 


\section{CIENCIAMATRIA}

Revista Interdisciplinaria de Humanidades, Educación, Ciencia y Tecnología

Año VI. Vol. VI. N¹0. Enero - Julio 2020

Hecho el depósito de ley: pp201602FA4721

ISSN-L: 2542-3029; ISSN: 2610-802X

Universidad Nacional Experimental Francisco de Miranda (UNEFM). Santa Ana de Coro. Venezuela

Maryuri Elizabeth Morejón Santistevan

crecimiento responsable $20 \%$, deficiente crecimiento responsable $13 \%$ y muy deficiente crecimiento responsable el $7 \%$

Satisfacción del cliente

Cuadro 2

Excelente 5
Buen 4

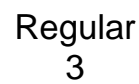

3
Deficiente 2
Muy Deficiente 1

\begin{tabular}{cccccccccc} 
FA & $\%$ & FA & $\%$ & FA & $\%$ & FA & $\%$ & FA & $\%$ \\
237 & 33 & 127 & 27 & 60 & 20 & 65 & 13 & 27 & 7 \\
\hline
\end{tabular}

La alternativa excelente satisfacción del cliente, posee una representación estadística de $33 \%$, en cuanto a la opción buena satisfacción del cliente, obtuvo el $27 \%$, regular satisfacción del cliente $20 \%$, deficiente satisfacción del cliente $13 \%$ muy deficiente satisfacción del cliente el $7 \%$

\section{Compromiso social}

Cuadro 3

Excelente

5

\section{Buen \\ 4}

$\underset{3}{\text { Regular }}$
Deficiente

2

Muy Deficiente

1

\begin{tabular}{cccccccccc}
\hline FA & $\%$ & FA & $\%$ & FA & $\%$ & FA & $\%$ & FA & $\%$ \\
93 & 38 & 197 & 28 & 145 & 11 & 54 & 5 & 27 & 18 \\
\hline
\end{tabular}

La alternativa excelente compromiso social, posee una representación estadística de $38 \%$, en cuanto a la opción buen compromiso social, obtuvo el $28 \%$, regular compromiso social $11 \%$, deficiente compromiso social $5 \%$ muy deficiente compromiso social el $18 \%$ 
CIENCIAMATRIA

Revista Interdisciplinaria de Humanidades, Educación, Ciencia y Tecnología

Año VI. Vol. VI. N¹0. Enero - Julio 2020

Hecho el depósito de ley: pp201602FA4721

ISSN-L: 2542-3029; ISSN: 2610-802X

Universidad Nacional Experimental Francisco de Miranda (UNEFM). Santa Ana de Coro. Venezuela

Maryuri Elizabeth Morejón Santistevan

\section{Compromiso ambiental}

Cuadro 4

\begin{tabular}{|c|c|c|c|c|c|c|c|c|c|}
\hline \multicolumn{2}{|c|}{$\begin{array}{c}\text { Excelente } \\
5\end{array}$} & \multicolumn{2}{|c|}{$\begin{array}{c}\text { Buen } \\
4\end{array}$} & \multicolumn{2}{|c|}{$\begin{array}{c}\text { Regular } \\
3\end{array}$} & \multicolumn{2}{|c|}{$\begin{array}{l}\text { Deficiente } \\
2\end{array}$} & \multicolumn{2}{|c|}{$\begin{array}{c}\text { Muy Deficiente } \\
1\end{array}$} \\
\hline FA & $\%$ & FA & $\%$ & FA & $\%$ & FA & $\%$ & FA & $\%$ \\
\hline 57 & 11 & 328 & 64 & 23 & 13 & 69 & 8 & 39 & 11 \\
\hline
\end{tabular}

La alternativa excelente compromiso ambiental, posee una representación estadística de $11 \%$, en cuanto a la opción buen compromiso ambiental, obtuvo el $64 \%$, regular compromiso ambiental $13 \%$, deficiente compromiso ambiental $8 \%$ muy deficiente compromiso ambiental el $11 \%$

\section{DISCUSIÓN}

Se analizó la empresa objeto de estudio en base a cuatro indicadores que conforman su filosofía de gestión: 1. Crecimiento responsable. 2. Satisfacción del cliente. 2. Compromiso social. 4. Compromiso ambiental, lo cual permite conocer la incidencia sobre la gestión pública, en ese sentido: Aguilar López (2015), en relación al crecimiento responsable, señala que:

La responsabilidad social corporativa empieza a dejar de ser un elemento decorativo, algo que se exhibe en la fachada para hacer que la empresa ofrezca una apariencia mejor. Este enfoque arcaico es remplazado por un responsabilidad tangible en todas las dimensiones de la empresa: sus procesos de negocio, la manera en la que se relaciona con todos los grupos de interés (clientes, proveedores...), etc. Las empresas socialmente responsables no son las que lo proclaman sino las que lo practican y no desde una dimensión colateral de su actividad sino desde su core, desde su núcleo. Para saber si una empresa es responsable lo que hay que mirar no es qué partida de sus presupuestos se dedica por ejemplo a acciones sociales o ambientales, sino el nivel de compromiso de cada una de las relaciones que esa organización establece con sus stakeholders.

Los resultados evidencian que la empresa objeto de estudio, ha interrelacionado de modo efectivo el concepto de crecimiento responsable en comparativa con lo planteado por el autor, así existe concordancia entre lo teórico y realidad social. 


\section{CIENCIAMATRIA}

Revista Interdisciplinaria de Humanidades, Educación, Ciencia y Tecnología

Año VI. Vol. VI. N¹0. Enero - Julio 2020

Hecho el depósito de ley: pp201602FA4721

ISSN-L: 2542-3029; ISSN: 2610-802X

Universidad Nacional Experimental Francisco de Miranda (UNEFM). Santa Ana de Coro. Venezuela

Maryuri Elizabeth Morejón Santistevan

En relación a la satisfacción del cliente, los resultados coinciden con Aldana \& Piña (2018), quienes destacan que una empresa debe cubrir las expectativas del cliente para que este se encuentre satisfecho, perfil que es recurrente a la empresa privada, cuando este se encuentra en la pública, se genera un principio de reciprocidad que permite proyectar una buena gestión pública.

En cuanto al compromiso social, los resultados concuerdan con Antelo \& Robaina (2015), quienes sostienen que "la responsabilidad social empresarial es el compromiso continuo de contribuir al desarrollo económico sostenible, mejorando la calidad de vida de los empleados y sus familias, así como la de la comunidad local y de la sociedad en general" (p.59), de ese modo, se vislumbra un contacto tangible de las políticas de la empresa con generar un compromiso social eficaz, según las perspectiva de la NGP. Por otro lado, el compromiso ambiental, en función de los resultados obtenidos, se muestra como satisfactorio, en concordancia López Echegarai (2019), por lo tanto, la empresa según la percepción de los encuestados, cumple con el compromiso ambiental de generar la protección sostenible del medio, lo cual permite generar la posibilidad de promover un acercamiento con la comunidad desde una óptica sostenible y responsable.

\section{CONCLUSIONES}

La Corporación nacional de telecomunicaciones - CNT EP, al ser evaluada según indicadores presentados en el documento "memoria de sostenibilidad 2017", teniendo para tal fin: 1. Crecimiento responsable. 2. Satisfacción del cliente. 2. Compromiso social. 4. Compromiso ambiental; de ese modo, en función de los resultados obtenidos de la aplicación de la encuesta, se considera que esta empresa cumple favorablemente con los postulados expuesto en el documento señalado, lo cual concuerda con teóricos relacionados al tema, generándose una concordancia que permite deducir que la empresa se encuentra desarrollándose en conformidad con los postulados de su filosofía de gestión y con la nueva gestión pública (NGP), lo cual permite proyectar el funcionamiento de una empresa pública en similitud de las operaciones de una privada, 
CIENCIAMATRIA

Revista Interdisciplinaria de Humanidades, Educación, Ciencia y Tecnología

Año VI. Vol. VI. N¹0. Enero - Julio 2020

Hecho el depósito de ley: pp201602FA4721

ISSN-L: 2542-3029; ISSN: 2610-802X

Universidad Nacional Experimental Francisco de Miranda (UNEFM). Santa Ana de Coro. Venezuela

Maryuri Elizabeth Morejón Santistevan

siendo esto un beneficio para la sociedad, observándose un eficiente aporte a la gestión pública del Ecuador.

\section{REFERENCIAS CONSULTADAS}

1. Aldana, J., \& Piña, J. (2018). Calidad del servicio prestado al cliente por los instructores de gimnasios. Revista Arbitrada Interdisciplinaria Koinonía, 2(3), 172197. Recuperado de http://fundacionkoinonia.com.ve/ojs/index.php/revistakoinonia/article/view/59/46

2. Arias, F. (2009). El Proyecto de Investigación. Guía para su elaboración. Caracas: Epísteme. Quinta Edición.

3. Aguilar López, J. (2015). Crecimiento responsable. Recuperado de http://www.eexcellence.es/index.php/entrevistas/coaching-empresarial/comohacer-crecer-las-empresas-desde-bases-solidas-y-sostenibles

4. Antelo, Y., y Robaina, D. (2015). Análisis de la Responsabilidad Social Empresarial basado en un modelo de Lógica Difusa Compensatoria. Ingeniería Industrial, 36(1), pp. 58-69. Recuperado de http://scielo.sld.cu/scielo.php?script=sci arttext\&pid=S1815$\underline{59362015000100007 \& \operatorname{lng}=e s \& t \operatorname{lng}=\mathrm{es}}$

5. Bedón, G. (s/f). La gestión pública tiene como referente al buen vivir. Recuperado de http://www.planificacion.gob.ec/la-gestion-publica-tiene-como-referente-albuen-vivir/

6. Caamaño Alegre, J. (2002). La modernización del sistema presupuestario de los EEUU bajo la era Clinton: balance de ocho años de reformas. Revista de Derecho Financiero y Hacienda Pública. LII (263): 175 - 245

7. Corporación nacional de telecomunicaciones - CNT EP (2017). Memoria de sostenibilidad 2017. Recuperado de https://corporativo.cnt.gob.ec/wpcontent/uploads/2019/01/memoriaSos2017.pdf

8. Hernández, Fernández y Baptista (2008). Metodología de la investigación. México, Mc Graw Hill Hispanoamericana. Hill Internacional

9. Lickert, R. (2000) Archives of Psychology d'attitudes avec excelled type Citado por Chiavenatto I (2000) Edit. McGraw Hill. México. 
Universidad Nacional Experimental Francisco de Miranda (UNEFM). Santa Ana de Coro. Venezuela

10. López Echegarai, M. (2019). Política de sustentabilidad ambiental. Avances en el contexto universitario. Revista Arbitrada Interdisciplinaria Koinonía, 4(7), 59-87. doi:http://dx.doi.org/10.35381/r.k.v4i7.194

11. Pichardo Pagaza, Ignacio (2004). Modernización administrativa. Propuesta para una reforma inaplazable. El Colegio Mexiquense, A.C. Facultad de Ciencias Políticas y Sociales, UNAM, ZINACANTEPEC, México, 391 pp., ISBN: 970-669063-8.

12. Pliscoff -Varas, Cristian. (2017). Implementando la nueva gestión pública: problemas y desafíos a la ética pública. El caso chileno. Convergencia, 24(73), 141-164. Recuperado

de http://www.scielo.org.mx/scielo.php?script=sci arttext\&pid=S1405$14352017000100141 \& \operatorname{lng}=$ es\&tlng=es.

13. Schröder, P. (2018). Nueva Gestión Pública: Aportes para el buen gobierno. Recuperado de http://relial.org/uploads/biblioteca/44dbee76837e79a6c07bb8219d021843.pdf

14. Tamayo y Tamayo (2009). El proceso de investigación científica. Limusa. México.

\section{REFERENCIAS CONSULTADAS}

1. Aldana, J., \& Piña, J. (2018). Quality of service provided to the client by gym instructors. Interdisciplinary Arbitrated Review Koinonía, 2 (3), 172-197. Recovered from http://fundacionkoinonia.com.ve/ojs/index.php/revistakoinonia/article/view/59/46

2. Arias, F. (2009). The Research Project Guide for its elaboration. Caracas: Epistle me. Fifth edition.

3. Aguilar López, J. (2015). Responsible growth Recovered from http://www.eexcellence.es/index.php/entrevistas/coaching-empresarial/comohacer-crecer-las-empresas-desde-bases-solidas-y-sostenibles

4. Antelo, Y., and Robaina, D. (2015). Analysis of Corporate Social Responsibility based on a model of Compensatory Fuzzy Logic. Industrial Engineering, 36 (1), pp. 58-69.

Retrieved from http://scielo.sld.cu/scielo.php?script=sci arttext\&pid=S1815$\underline{59362015000100007 \& \operatorname{lng}=\text { en\&tlng=en }}$ 
CIENCIAMATRIA

Revista Interdisciplinaria de Humanidades, Educación, Ciencia y Tecnología

Año VI. Vol. VI. N¹0. Enero - Julio 2020

Hecho el depósito de ley: pp201602FA4721

ISSN-L: 2542-3029; ISSN: 2610-802X

Universidad Nacional Experimental Francisco de Miranda (UNEFM). Santa Ana de Coro. Venezuela

Maryuri Elizabeth Morejón Santistevan

5. Bedón, G. (s / f). Public management is based on good living. Recovered from http://www.planificacion.gob.ec/la-gestion-publica-tiene-como-referente-al-buenvivir/

6. Caamaño Alegre, J. (2002). The modernization of the US budget system under the Clinton era: balance of eight years of reforms. Journal of Financial Law and Public Finance. LII (263): 175-245

7. National telecommunications corporation - CNT EP (2017). Sustainability report $2017 . \quad$ Recovered from https://corporativo.cnt.gob.ec/wpcontent/uploads/2019/01/memoriaSos2017.pdf

8. Hernández, Fernández and Baptista (2008). Investigation methodology. Mexico, Mc Graw Hill Hispanic American. Hill International

9. Lickert, R. (2000) Archives of Psychology d'attitudes avec excelled type Cited by Chiavenatto I (2000) Edit. McGraw Hill Mexico.

10. López Echegarai, M. (2019). Environmental sustainability policy. Advances in the university context. Interdisciplinary Arbitrated Review Koinonía, 4 (7), 59-87. doi: http: //dx.doi.org/10.35381/r.k.v4i7.194

11. Pichardo Pagaza, Ignacio (2004). Administrative modernization Proposal for an unplayable reform. The Colegio Mexiquense, A.C. Faculty of Political and Social Sciences, UNAM, ZINACANTEPEC, Mexico, 391 pp., ISBN: 970-669-063-8.

12. Pliscoff -Varas, Cristian. (2017). Implementing the new public management: problems and challenges to public ethics. The Chilean case. Convergence, 24 (73), 141-164. Retrieved http://www.scielo.org.mx/scielo.php?script=sci arttext\&pid=S1405$14352017000100141 \&$ Ing=en\&tlng=en.

13. Schröder, P. (2018). New Public Management: Contributions to good governance. Retrieved

from http://relial.org/uploads/biblioteca/44dbee76837e79a6c07bb8219d021843.pdf

14. Tamayo and Tamayo (2009). The process of scientific research. Limusa Mexico.

(C2020 por el autor. Este artículo es de acceso abierto y distribuido según los términos y condiciones de la licencia Creative Commons Atribución-NoComercial-Compartirlgual 4.0 Internacional (CC BY-NC-SA 4.0) (https://creativecommons.org/licenses/by-nc-sa/4.0/) 